\title{
Design of Novel Online Access and Control Interface for Remote Experiment on DC Drives
}

\author{
doi:10.3991/ijoe.v5i2.788 \\ Jagadeesh Chandra A.P, and R.D Sudhaker Samuel \\ S.J College of Engineering, Mysore, India
}

\begin{abstract}
Internet has revolutionized the way in which information is delivered. Laboratory based courses play an important role in technical education. Automation is changing the nature of these laboratories and the system designer's focus on Internet accessed experiments owing to the availability of several tools to integrate electronic and mechanical hardware with the World Wide Web. Standalone approaches in remote learning have grown tremendously in the recent years. One of the important components in remote experimentation is the integration of Virtual Instruments to perform real hardware tasks in near real-time. The paper describes a web interface to the electrical hardware and integration of LabVIEW Virtual Instruments to the remote access and control of DC Drives. Customized electrical hardware serves as the web interface, supporting various features to remotely control and measure the parameters of the electrical machine. Novel techniques have been used to interface a low power data acquisition system with the DC machine driven by the electric mains. The system uses the client-server architecture to access the web page of the Virtual Instruments through web browser. The developed system imitates the real control of experiment hardware, but being operated remotely through Internet.
\end{abstract}

Index Terms-Data Acquisition, DC Drive, Virtual Instruments, Web-server.

\section{INTRODUCTION}

Online access and control of a complex hardware or equipment is a compelling issue in remote laboratories and industrial applications. Developments in computer networking have provided new possibilities and new challenges for designing and deploying remote engineering applications through a commonly available web browser. Recent years have seen tremendous innovation in integrating remote access hardware or equipment for Internet based learning. The demands on a good co-operative learning technology include friendly access, near real-time operation, visual access and efficient utilization of the available bandwidth. Making use of advances in information technology is an economical and effective approach in laboratory based course delivery systems.

One of the important applications of remote engineering is the development of e-learning methods with remote laboratories [6]. The complex tasks in electrical engineering cannot be successfully taught without the support from new technologies. The challenges in remote experimentation are that to give real world experience with the control objects similar to the manual operation [12]. Researchers must carefully isolate and find the factors which influence the remote laboratory in determining the effectiveness in education system [5].

The important components of the remote experiments are the Virtual Instrumentation (VI) tools integrated to perform real hardware tasks in real time. At the core of the remote laboratory lies the concept of distance learning, where tools and equipment used in the learning process are distributed over several geographical locations [14]. Important ingredients in technical training are practical laboratory activities, recognized as an efficient approach to understand the theoretical concepts as well as to draw vital experimental data from remote locations for analysis and design. There are standard technology tools with low power data acquisition is available for remote access of electronic hardware, which can be used with the simple control applications or remote laboratory experiments [10]. The same techniques are used to demonstrate remote embedded control applications with additional software to handle the programming of microcontroller remotely [9]. The development of remote laboratory facilities on power circuits by placing limits on electrical parameters for safety reasons and implemented using simulation tools for prelaboratory investigations [11]. The implementation of effective remote hardware experimentation system is possible with the integration of custom designed tools and the standard computing tools such as Matlab/Simulink/Control Desk [2]. Use of DSP based rapid control prototyping (RCP) systems enables quick and easy development of different remote control experiments but the operation is limited to low power applications [13]. Due to the limited operating range of voltage and current, the web-interface to electronic hardware is developed by standard data acquisition (DAQ) systems [16]. Customized modules in electrical hardware experiments provide vital features with ease of operation offering designers the flexibility to make changes based on the user requirement to achieve the desired objective.

The design concepts described in this paper is suitable for remote experiments on high power electrical devices and circuits, which operates with $0-230 \mathrm{~V}$ input and up to 20A of load current. The proposed architecture illustrates the use of standard DAQ systems in web based real-time access and control of high power remote electrical applications. Sophisticated interface structure is designed using LabVIEW to address the safe and user friendly interface with precision control and measurement techniques. The live video of the remote laboratory is embedded with the HTML page and the features to control and measure the experimental parameters are displayed on the interactive user interface developed using LabVIEW.

The system architecture is based on client/server model where the communication uses the standard TCP/IP proto- 


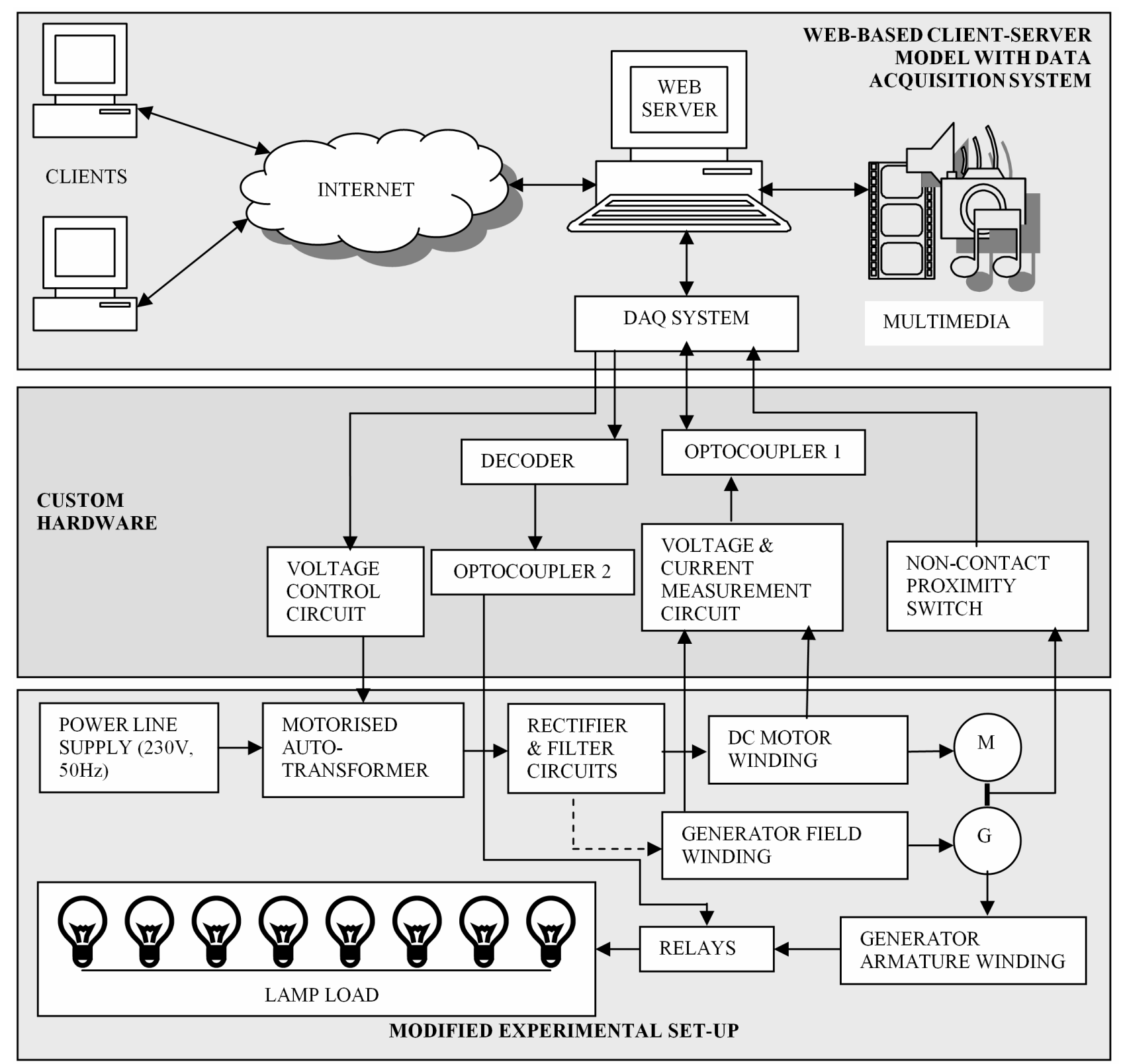

Figure 1. System architecture for remote laboratory experiment on DC Drives

col. The standard data acquisition system consisting of PCI6251 and Electronics Laboratory Virtual Instrumentation Suite (ELVIS) from National Instruments, and the customized hardware is used to control various functions of electrical machine. The LabVIEW built-in web server handles the secured access of clients with the required amount of control to the remote device. A web camera with audio inputs interfaced with the web server transmits audio and video signals to the client in real time enabling the remote user to experience the real environment.

The paper is organized as follows: Section II introduces the system architecture, which describes the hardware and software elements used in the development of remote experimentation on DC drives, Section III presents the design of interface between the DAQ and the power circuit along with some novel design techniques used in the control and measurement while Section IV describes the design of LabVIEW Virtual Instruments (VI) and a multimedia tool to support online collaborative learning and finally the conclusion.

\section{SyStEM ARCHITECTURE}

This section presents the structure of the remote experimentation system on DC drives. The architecture is developed in modular fashion so that each module can be changed in an independent manner. The architecture consists of a web server, data acquisition system, custom designed circuits, DC motor-generator combination, and the variable load. The architecture is shown in Fig. 1.

The architecture is divided in to three sections.

a) Client-server model and standard DAQ system

b) Custom Hardware

c) Modified Experimental Set-up

The web server is the central entity in establishing the communication between the remote clients and the electrical hardware. The web server is primarily used for 
data acquisition and control operations. The graphical programming tool LabVIEW with its intuitive user interface provides the development platform to control the system operation remotely and display the measured parameters in real-time [1]. It is used with the third party software, making it more attractive for remote access applications. The other reason for choosing LabVIEW is for its built-in server facility that can be utilized to publish the Graphical User Interface (GUI) for web based experiments. The graphical interface to the client side is developed by creating HTML page of the control panel, which includes the visual display of control objects and virtual meters to display the measured electrical parameters of the experiment. The multimedia features are used to experience the real environment of the laboratory and facilitate the collaborative access between the server and the remote user. The custom hardware consists of various elements to interface between the web server and the experimental hardware.

The need for separate signal conditioning arises due to the experiment operating range, which is high (Voltage: 0230 V, Current: 0-20A) and the ready data acquisition systems cannot handle high power devices such as $1.5 \mathrm{~kW}$ motor and $750 \mathrm{~W}$ generator, used in the experiment. The input voltage to the motor and generator is derived from the ac mains and rectifiers. The system is designed analogous to manual input voltage control using autotransformers, but operated using the computer keyboard or mouse. The control is automated using the motorized autotransformer and custom logic circuitry operated by the DAQ output commands generated from the web server. There are independent signal path and control elements for the inputs applied to motor and generator windings. The dotted line in Fig. 1 indicates that the generator field winding is also receives the input from rectifier with similar constructs as used for motor windings. The decoder selects the variable load by switching the relays connected in the output path of the DC drive. Hence the experiment can be performed with different load conditions.

The circuits to measure voltage and current of the experiment are directly connected to the armature and field windings of the DC drive. These circuits are designed to perform the down conversion of electrical signals from $0-230 \mathrm{~V}$ DC to $0-10 \mathrm{~V} \mathrm{DC}$ while maintaining the linearity in operation. The custom built circuits are also used to interface motor/generator windings with DAQ to measure the various electrical parameters of the experiment. The DAQ system supports input/output (I/O) ports for both analog and digital signal interface. The I/O ports are selected for the interface based on the experiment requirements. The DAQ system and the external power devices are completely isolated by the optocouplers. The speed of the DC drive is measured using non-contact proximity switch, where the digital pulses are generated for each rotation of the shaft and counting the number of pulses per minute gives the RPM.

The final experimental set-up on DC drives is similar to the motor control circuits used in the electrical engineering laboratory. It has the motorized autotransformer with $12 \mathrm{~V}$ DC servomotor controls the input voltage to the rectifiers. The servomotor is controlled by the digital outputs of the DAQ, which positions the wiper of the autotransformer to produce the desired output.

The major contributions of this development are,
- Customized design of the input voltage control variations $0-230 \mathrm{~V}$ achieved remotely in steps of $+/-$ $1 \mathrm{~V}$ by operating the digital switches using computer keyboard or mouse and

- the measurement range, Voltage: $0-230 \mathrm{~V}$, Current: 0-20A being displayed on the web page by maintaining perfect isolation between server and the electrical hardware.

The LabVIEW based GUIs are subsequently transformed to dynamic web page and stored in the server. These dynamic web pages are linked with the web application that hosts the remote experiments. The clients can access the experiment by accessing the GUI along with the multimedia features. The web camera streaming software WebcamXP5 is used for broadcasting audio and video over the web. A client PC should have Internet explorer and LabVIEW run-time engine (freeware) to perform the task.

\section{WEB-INTERFACE AND EXPERIMENT HARDWARE}

The first step towards the development of web based laboratory facility is to establish an interface between the server and the experiments. The custom designed hardware is the electric circuits used to interface the experimental set-up with the standard DAQ system in the web server. The DAQ system consists of National Instruments PCI6251 and ELVIS workstation, which supports 12 channels of analog inputs (AI0-AI11) in Referenced Single Ended (RSE) mode, 2 channels of analog outputs (AO0-AO1) with 16 bit resolution and 8 independent channels of digital inputs/outputs (I/Os) (Input: DI0-DI7, Output: DO0-DO7) used by NI ELVIS digital bus writer SFP (Soft Front Panel). The DAQ PCI6251 supports 16 PFI (Programmable Function I/O, PFI 0-15) or Static digital I/O and two counters/timers with the resolution of 32 bits. The PFI lines are individually programmable as input or output and the counter/timer outputs of DAQ can also be routed to each PFI I/Os. PFI 9 is the default input terminal for low frequency measurement using counter 0 , which is used to measure the RPM of motor-generator combination using non contact proximity switch. The analog I/O's are operated at 0 to $+/-10 \mathrm{~V}$ and digital $\mathrm{I} / \mathrm{Os}$ are TTL compatible with 8 bit resolution [16].

\section{A. Input Voltage Control Interface}

Remote control of input voltage to the DC drives is achieved using the motorized autotransformer with servo motor is controlled by the digital output commands from the web server. The basic electrical diagram of the servo control mechanism is shown in Figure 2. The motorized

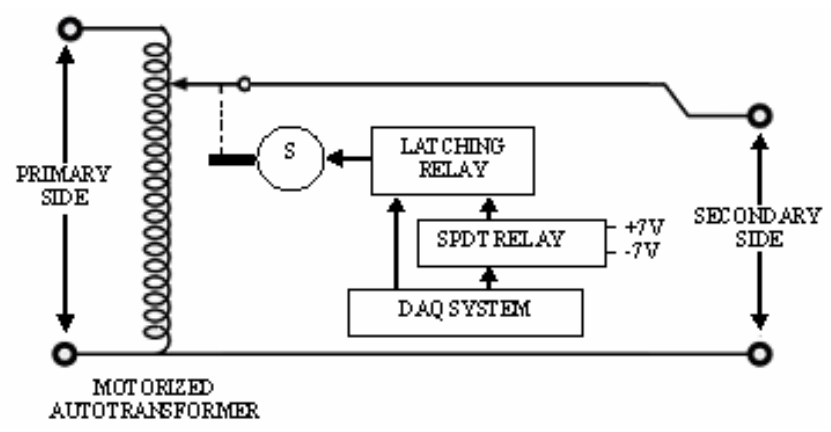

Figure 2. Input voltage control interface with DAQ system. 
auto transformer controls the input to the rectifier circuit. The user can set the position of the autotransformer wiper by operating the LabVIEW virtual instruments either through mouse or keyboard, but accurate settings can be made using keyboard control.

The hardware consists of motorized variable output auto transformer with $12 \mathrm{~V}$ DC servo motor, Single Pole Double Throw (SPDT) relay, latching relay and data acquisition system. The digital control signals from the data acquisition system controls the SPDT and latching relays to operate the servomotor with $+/-7$ Volts. The connected polarity of the DC supply decides the direction of rotation of the servo motor, which moves the autotransformer wiper towards the increment or decrement of secondary voltage.

The DC servomotor is operated with $+/-7 \mathrm{~V} \mathrm{DC}$, which gives the secondary side voltage control in steps of $+/-1 \mathrm{~V}$. Hence the output of the autotransformer is digitally controlled for the complete range of $0-230 \mathrm{~V}, 50 \mathrm{~Hz}, \mathrm{AC}$ signal in steps of $1 \mathrm{~V}$. The Boolean Control features of LabVIEW are used to generate the digital stimulus to the control circuit shown in Fig.2.

The ELVIS workstation supports 8 digital output ports; hence the PFI1 and PFI2 are configured to operate as digital outputs to meet the required number of digital control ports in the experiment system. The digital outputs DO1 to DO6 are used to control the input voltages to the field and armature windings of motor and field windings of generator. Pair of digital outputs is used to control a motorized auto-transformer, which supplies input voltages to the corresponding windings of the DC drive. The armature winding of the generator is interfaced with 8 lamp loads, which are selected by 3 -input decoder. Table 1 describes the interface between the DAQ system and control hardware.

TABLE I.

OUTPUT PORT INTERFACE OF DAQ SYSTEM

\begin{tabular}{|c|c|c|c|}
\hline $\begin{array}{c}\text { SI. } \\
\text { No }\end{array}$ & $\begin{array}{c}\text { ELVIS } \\
\text { Interface }\end{array}$ & Control Action & $\begin{array}{c}\text { Computer } \\
\text { Key }\end{array}$ \\
\hline 1 & DO0 & ON/OFF & F1 \\
\hline 2 & DO1, DO2 & $\begin{array}{c}\text { Motor Field } \\
\text { Voltage Control }\end{array}$ & F2, F3 \\
\hline 3 & DO3, DO4 & $\begin{array}{c}\text { Motor Armature } \\
\text { Voltage Control }\end{array}$ & F4, F5 \\
\hline 4 & DO5, DO6, DO7 & Decoder Inputs & F6, F7, F8 \\
\hline 5 & PFI1, PFI2 & $\begin{array}{c}\text { Generator Field } \\
\text { Voltage Control }\end{array}$ & F9, F10 \\
\hline
\end{tabular}

\section{B. Isolation and Down Conversion Logic}

The electrical features of the remote experiment such as DC voltage $0-230 \mathrm{~V}, 0-20 \mathrm{~A}$ load current, are recorded online with complete electrical isolation. The voltage and current down conversions are performed separately, but with similar blocks as shown in Fig 3.

The various components constructs voltage down conversion is shown in Fig. 3(a). The attenuator reduces the amplitude of the signal from $0-230 \mathrm{~V}$ to $0-10 \mathrm{~V}$. Isolation is a problem when larger $\mathrm{AC}$ and $\mathrm{DC}$ voltages need to be interfaced to standard DAQ circuits. The simple solution to this problem is to use optocoupler. The voltage to frequency $(\mathrm{V} / \mathrm{F})$ converter outputs $0-10 \mathrm{KHz}$ rectangular pulses for the input range of $0-10 \mathrm{~V}$ DC. The optocoupler outputs these pulses to frequency to voltage (F/V) converter which reconverts back to $0-10 \mathrm{~V}$ from 0 $10 \mathrm{KHz}$ pulses. Hence the output of $\mathrm{F} / \mathrm{V}$ converter is electrical isolated, down-converted and linearly proportional to the experiment operating range $0-230 \mathrm{~V}$ DC. The low pass filter (LPF) outputs the DC signal without ripples present in it. The analog inputs AI0-AI7 are used to measure the experiment parameters and interface details are given in Table 2.

The current measurement follows the same principle with current to voltage $(\mathrm{I} / \mathrm{V})$ converter placed at the first stage of the signal path shown in Fig. 3(b).

TABLE II.

INPUT PORT INTERFACE OF DAQ SYSTEM

\begin{tabular}{|c|c|c|}
\hline SI. No & $\begin{array}{c}\text { ELVIS } \\
\text { Interface }\end{array}$ & Measurement Using VI \\
\hline 1 & AI0 & Motor Field Voltage \\
\hline 2 & AI1 & Motor Field Current \\
\hline 3 & AI2 & Motor Armature Voltage \\
\hline 4 & AI3 & Motor Armature Current \\
\hline 5 & AI4 & Generator Field Voltage \\
\hline 6 & AI5 & Generator Field Current \\
\hline 7 & AI6 & Generator Armature (Load) Current \\
\hline 8 & AI7 &
\end{tabular}

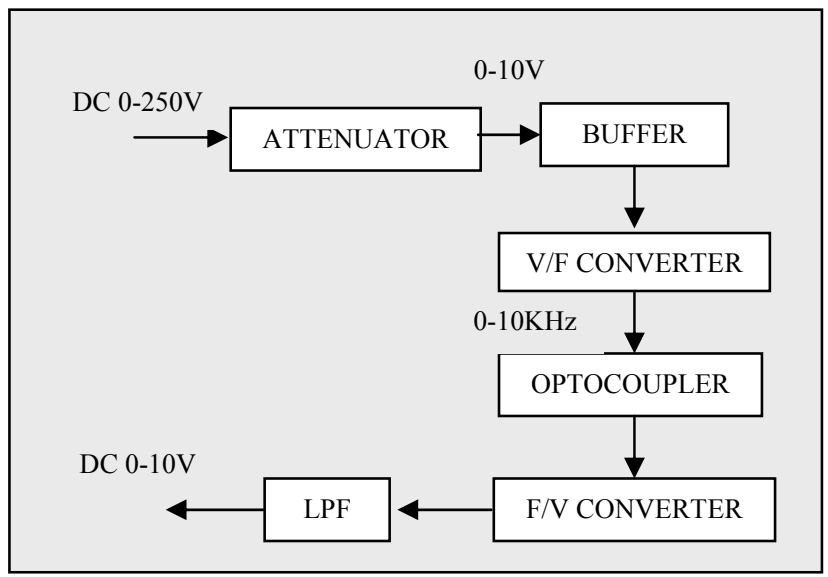

(a)



(b)

Figure 3. Block diagram of Isolation and Down-conversion logic. (a) Voltage down-conversion (b) Current down-conversion 
The small voltage proportional to current is suitably processed through the buffer and voltage amplifier to obtain the signal suitable for data acquisition system. This signal is optically isolated and fed to the input channel of DAQ system.

\section{Server Configuration}

In order to publish LabVIEW based Virtual Instruments (VI) on the web page, the server must be enabled through the configuration settings located with in the LabVIEW tools. The web publishing tool available in the configuration section is used to locate the HTML files out of the front panel of VI. Within the configuration settings the root directory, HTTP port, the time out period is set and the server is enabled. For all there are default settings and the HTML files are saved with in the LabVIEW www directory (eg: C: $\backslash \backslash$ Program Files $\backslash$ National Instruments $\backslash$ LabVIEW 8.5 $\backslash$ www). The user may access any of the HTML pages saved at root directory of web server. The VI developed for the remote experiment is mentioned in the VI access list and IP addresses of users are mentioned in browser access list of server configuration. The user may access any of the HTML pages saved at the root directory of web server. The client side uses the "LabVIEW run-time engine" which is about $24 \mathrm{MB}$, freely downloadable software, to execute the commands in remote environment. The web server is quite independent from client's operating system. The run-time engine is available for every operating system and could be used with every standard web browser [8].

\section{ONLINE ACCESSIBLE VIRTUAL INSTRUMENTS AND COLLABORATIVE LEARNING}

The main objective of remote experimentation facility is to embed flexibility in laboratory based learning practices by extending its operation by allowing accessibility to geographically dispersed places. The collaborative learning tools are embedded with remote laboratory infrastructure to create the online learning facility to more realistic [3]. The other benefits are the remote laboratory features can also be used with regular hands-on laboratory and virtual (simulation) laboratory in a mixed reality infrastructure to increase the efficiency in teaching and learning [4]. The basic building blocks in the implementation of remote experiment on DC Drives are LabVIEW based Virtual Instruments, which interfaces the real hardware with networked computer. It is seen in real hands-on laboratory that the use of real instruments for control and measurements are mediated through computers. The successful implementation of web based experimentation system is possible only when there is no much difference between the user operation with real instruments and the virtual instruments used in remote laboratories.

\section{A. LabVIEW Virtual Instruments}

The remote experiment system developed using LabVIEW offers several methods for remote access using TCP/IP protocol without additional programming. The LabVIEW programs are called Virtual Instruments because their appearance and easy operation imitate the real instruments. LabVIEW program consists of an interactive user interface called front panel and the source code in the form of block diagram. It includes extensive libraries of functions such as data acquisitions, data analysis, data presentation and storage. The benefits of LabVIEW web server are: the shortest development time, best user handling and client run on Windows, Linux, Mac and with nearly every browser. Thus, the web based laboratory is designed with minimum software requirements [7]. The LabVIEW front panel developed to control and measure the parameters of the remote hardware experiment is shown in Fig 4.

The LabVIEW graphical code is developed to read the electrical parameters of the hardware experiment. The DAQ system receives all the electrical parameters in the range of $0-10 \mathrm{~V}$, which is converted to read the actual value of the experiment using a suitable scaling factor in graphical code. For example the motor armature current is in the range of $0-20 \mathrm{~A}$, but DAQ receives it as $0-10 \mathrm{~V} \mathrm{DC}$ value at input port AI3. It uses a scaling factor of 2 to convert the DAQ range in to the actual value of the experiment reading. Similarly the real values of other parameters are also measured.

There are digitally operated Boolean controls are shown in Fig. 4, generates control signals to the motorized autotransformer present with each input voltage control section of the motor-generator windings. The keyboard shortcuts are used with each control actions, where the signal latches to the hardware when the key is operated. For example, when the Key F2 is latched (Boolean Control ON); the input voltage of motor field winding is set to vary in increasing direction. Now Key F3 is operated to increase the voltage in steps of $1 \mathrm{~V}$. The operation of Key F3 with releasing Key F2 (Boolean Control OFF) results in decrement of motor field voltage in steps of 1V. Similarly the keys F4, F5 controls the motor armature voltage and F9, F10 controls generator field voltage. The other keys F6-F8 control the decoder inputs, which selects the 8 lamp loads connected to armature windings of the generator through relays. The Key F1 is used as main power control to the experiment. The keyboard shortcut feature for control functions is available with LabVIEW.

\section{B. Collaborative Learning Support}

The control interface facilitates to start the experiment, vary the input parameters, set the load, record the readings and stop the experiment. The multimedia features have been supported along with the control interface to provide the user to have real laboratory experience by accessing the live video window of the experiment hardware. The collaborative and supported learning environment is embedded in a web browser and navigated using a tab system developed in HTML. Functionally it has three distinct but interconnected sections comprised of learning support resources, i.e., remote experimentation facilities, collaborative working and communication tools. Collaborative learning environment provides opportunities to interact, practice and to understand the need of working. The Information and Communication Technology (ICT) tools support collaborative activity in elearning. The use of webcamXP software provides live video and audio, which can be published together with the LabVIEW front panel.

WebcamXP allows sharing camera images online by integrating with the preexisting and custom coded website. Thus the live video window is embedded with the front panel VIs allows the users to view the real experimental process using the images captured by single 


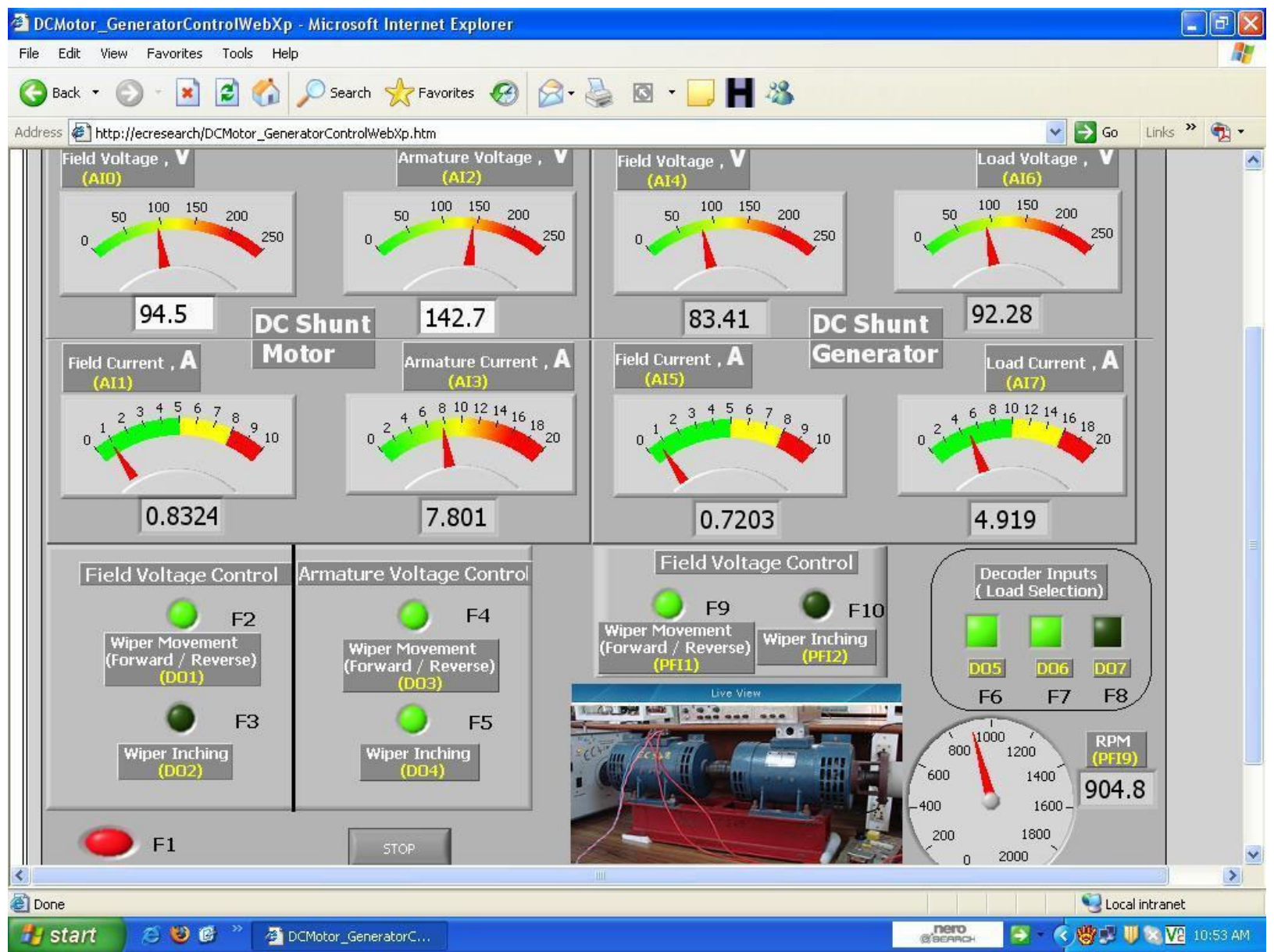

Figure 4. Web page of the front panel along with live view of experiment hardware

or several web cameras located in the laboratory. WebcamXP is a powerful webcam and IP cameras management and streaming software, which offer features to broadcast multiple video sources on the same computer [15]. The additional functions supported by webcamXP are audio transmission and live chat helps to set-up an online talking space between the client and server entities

WebcamXP allows sharing camera images online by integrating with the preexisting and custom coded website. Thus the live video window is embedded with the front panel VIs allows the users to view the real experimental process using the images captured by single or several web cameras located in the laboratory. WebcamXP is a powerful webcam and IP cameras management and streaming software, which offer features to broadcast multiple video sources on the same computer [15]. The additional functions supported by webcamXP are audio transmission and live chat helps to set-up an online talking space between the client and server entities.

\section{CONCLUSION}

Internet Technologies have opened a variety of new methodologies to enhance the experience of learning with expanding educational opportunities to a large pool of learners. Advancements is web technology facilitate remote engineering tasks in a truly near real-time online environment. Distance learning especially with remote laboratory facility contributes to online learning in all sector of engineering education around the world. In today's changing environment, web based courses offer flexibility in learning. The learner should be equipped with sufficient resources to access the facilities along with computer skills.

A novel interface and control technology has been presented to offer online experiments to power drives, which standard DAQ systems are unable to handle. Novel techniques have been presented to enhance the capacity of computer based virtual instruments to measure high range voltage and currents. This methodology creates an environment for remote access of applications that perform power system experiments remotely. The multimedia features are embedded with LabVIEW virtual instruments using webcamXP benefits with transmission of live video on to client's machine along with the control panel without any additional programming. The flexible and versatile design and implementation of the system is suitable for a wide range of experiments on DC drives.

\section{REFERENCES}

[1] M.B. Naumovic, D. Zivanovic. "Remote experiments in control engineering education laboratory", International Journal of Online Engineering (iJOE), Vol.4, No. 2, 2008, pp.48-53.

[2] C. Guerra Torres, J. de Leon Morales, A. Glumineau, D. Traore, R. Boisliveau. "Teleoperation of an experimental platform of electrical machines through the internet", International Journal of Online Engineering (iJOE), Vol. 4, No.1, 2008.

[3] MJ. Challaghan, J. Harkin, McGinnity, LP. Maguire. "Paradigms in Remote Experimentation", International Journal of Online Engineering (iJOE), Vol. 3, No. 4., 2007.

[4] Dieter Muller, F. Wilhelm Bruns, Heinz-Hermann Erbe, Bernd Robben, Yong-Ho Yoo. "Mixed Reality learning spaces for 
collaborative experimentation: A challenge for engineering education and training", International Journal of Online Engineering (iJOE) Vol.3, No. 4, 2007.

[5] Jing MA and Jeffrey V Nickerson. "Hands-on, simulated, and remote laboratories: A comparative literature review", ACM Computing Surveys, Vol.38, No.3, Article 7, 2006.

[6] Emi Ernest, Rafal Sztylka, Bartlomiej Ufnalski, Wlodzimierz Koczara, "Methods in teaching modern ac drives: inverter-fed motor system with internet-based remote control panel", Proceedings of 12th International Power Electronics and Motion Control Conference, Portoroz, Solvenia, 2006, pp.2130-2133.

[7] Adul K.M Azad. "Internet-based laboratory experiments: Exploring its potentials", Proceedings of International Conference on Engineering and Technology: Research-EducationEntrepreneurship (IJME-INTERTECH), Keen University, 2006.

[8] C. Mergl. "Comparison of remote labs in different technologies", iJOE International Journal of Online Engineering (iJOE), Vol.2 No.4, 2006.

[9] M.Gilibert, J.Picazo, M.Auer, A.Pester, J.Cusido, J.A. Ortega. "80C537 Microcontroller remote lab for e-learning teaching", iJOE International Journal of Online Engineering, vol.2, 2006

[10] Shuhui Li, Abrar A. Khan, "Applying IT tools to a laboratory course for measurement, analysis, and design of electric and electronic circuits" IEEE Transactions on Education, Vol.48, No.3, pp.520- 530, 2005. (doi:10.1109/TE.2005.852601)

[11] William Gerard Hurley, Chi Kwan Lee. "Development, implementation, and assessment of a web-based power electronics laboratory", IEEE Transactions on Education, Volume48, Issue 4, 2005, pp.567-573. (doi:10.1109/TE.2005.856147)

[12] Viliam Fedak, Pavol Bauer. "E-learning in education of electrical drives and power electronics: opportunities and challenges",
International Conference on Electrical Drives and Power Electronics (EDPE), Dubrovnik, Croatia, 2005.

[13] Darko Hercog, Bojan Gergic, Vojko Matko. "Remote Lab for Electric Drives", IEEE International Symposium on Industrial Electronics (ISIE), Dubrovnik, Croatia, 2005.

[14] Tan, K.K., T.H. Lee and F.M. Leu, "Development of a distant laboratory using LabVIEW", The International Journal of Engineering Education (iJOE), Vol.16, No.3, pp. 273-282, 2000.

[15] WebcamXP 5, User Manual, revision 1.3, Moonware Studios, 2008.

[16] NI Educational Laboratory Virtual Instrumentation Suite (NI ELVIS) User Manual, National Instruments, National Instruments Corporation, 2006.

\section{AUTHORS}

Jagadeesh Chandra A.P (apjchandra@yahoo.com) is with the Electronics and Communication Department of J.S.S. Research Foundation, Sri Jayachamarajendra College of Engineering, Mysore, India, on leave from Adichunchanagiri Institute of Technology, Chikmagalur, India.

R.D Sudhaker Samuel (sudhakersamuel@yahoo.com) is with the Electronics and Communication Department of Sri Jayachamarajendra College of Engineering, Mysore, India.

This work is supported by the Department of Science and Technology, Ministry of Science and Technology, Government of India under Grant SR/FST/ETI-033/2005.

Manuscript received 28 December 2008. Published as submitted by the authors. 